\title{
anatomy
}

\section{Welcome Address of the Programme President}

\author{
Anatomy 2010; 4 Suppl 1: 8 @ 2010 TSACA
}

As the scientific and programme president of $2^{\text {nd }}$ International Symposium of Clinical and applied Anatomy, held in Prague from $9^{\text {th }}$ to $11^{\text {th }}$ July, 2010, I am honoured to have this possibility to bid welcome you to this special occasion.

The organizers of any scientific meeting have usually two basic wishes. First, rich and good program, depending on the results of actu-

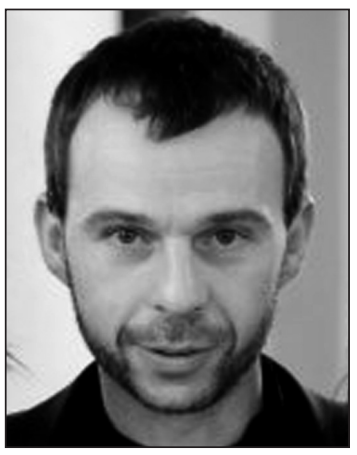

UNICA presents itself as an institution involved mainly into the education of postgraduate students and into the orientation close to the „research university“. At all five medical faculties of Charles University in Prague the teaching of anatomy has an old and good tradition. At the Third Faculty of Medicine, a new medical curriculum was introduced 13 years ago, oriental research activities of participants, and second, contentment of the participants with the social program, offering many direct personal and professional contacts of participants as well as rich mutual contacts with the local culture and history. The success of the symposium strictly depends on the fulfilling of both conditions mentioned above.

Last year we have the chance and honour to enjoy the very first International Symposium of Clinical and applied Anatomy, held in Novi Sad, Serbia, thanks to the enormous effort of Dragan Krivokuća, Mirela Erić and their team. The fact that the $2^{\text {nd }}$ ISCAA is organized this year in Prague, the capital of Czech Republic, give us the hope that also this time the conditions for a successful congress will be fulfilled. Prague is a seat of the venerable university, having now about 47,000 students, and has very intensive scientific and pedagogic contacts with more than 170 universities worldwide. Its position in Europe is therefore remarkable. The Charles University in Prague is also intensively engaged in the Bologna process activAssociate Professor David Kachlík, MD, PhD Programme President ities, and its memberships in the Coimbra Group and in the ed in the first two years on the integrated study of the basic medical disciplines, and later on more on the problem based learning and on the clinical rotations. The organizers of the ISCAA hope that their partial presentations will give all foreign participants good survey of the level, on which the anatomy is taught at our medical school, including the courses of the clinical anatomy.

It is really a very gratifying fact that this year Prague has become a center, where the anatomists from thirty countries of several continents can meet each other. It is our deep hope and wish that all participants will leave our capital with the best memories on good congress level, and on nice atmosphere and beauties romantic „golden Prague on the Vltava River“.

ISCAA 2010 Prague 